\title{
Displaying Image Neighborhood Hypergraphs Line-Graphs
}

\author{
S. Chastel, P. Colantoni, and A. Bretto \\ LIGIV - Université de Saint-Étienne \\ 3, rue Javelin Pagnon \\ BP 505 \\ F-42007 SAINT-ÉTIENNE Cedex 01 \\ chastel@vision.univ-st-etienne.fr
}

\begin{abstract}
Graph-based structures are commonplace in image processing. Our contribution in this article consists in giving hints representing a new modeling of digital images: image neighborhood hypergraphs. We give some results on the hyperedges coloring of them. We also describe techniques we used to display image neighborhood hypergraphs line-graphs. These techniques form the basis of a tool that allows the exploration of these structures. In addition, this tool can be used to visualize, explore and describe features of image regions of interest such as object edges or noise.
\end{abstract}

For nearly fifty years, image processing, image analysis, and computer vision are fields that have been intensively studied. Many various mathematical theories and techniques have been widely involved in these scientific fields: linear algebra, continuous optimization, statistics, combinatorics, to name a few. One of the main problematics in image analysis is the foundation of formal modelings of the digital image. In any scientific field, an efficient modeling should establish structural relationships between the different objects of that domain. A digital image may be considered as a union of elementary spatial and colorimetric units. Therefore, working on the organization of these components may be seen as a combinatorial problem, since combinatorics is the science that studies organization of discrete objects in mathematically formalized modelings. This is maybe why graph theory and associated techniques arose in image analysis and had been widely used by many researchers. An attractive approach is to develop a modeling technique based on a generalized graph theory: hypergraph theory. Hypergraph theory arose from the seminal work of C. BERGE in the early seventies [2]. In graph theory, one studies the binary relationships between elements. In hypergraph theory, the relationships between basics elements are generalized: elements in relation belong to a same set if they share a common property.

We recently proposed a modeling based on hypergraph theory [6]. Our first intentions in dealing with this modeling was to try to characterize regions of interest in a picture. That is why we tried to represent the image neighborhood hypergraph structure itself and this paper presents some results on this. Trying

A. Braquelaire, J.-O. Lachaud, and A. Vialard (Eds.): DGCI 2002, LNCS 2301, pp. 124-135 2002. (C) Springer-Verlag Berlin Heidelberg 2002 
to represent it in a bidimensional way in simply drawing and coloring the hyperedges, we will show that this might appear as an unappropriate representation of that modeling. A tridimensional representation of the INH line-graph appeared to be a better way to display them and is the objet of our third part.

\section{Theoretical Background}

Given a set $X$, we will denote by $X^{(2)}$ the set of unordered pairs of $X$. A graph $G\left[113\right.$ is an ordered pair $(X, E)$ such that $E$ is a subset of $X^{(2)}$. The size of $G$ is the cardinality of $X$. Elements of $X$ are called vertices, those of $E$ edges. If $e=\{x, y\}$ is an edge of $G, x$ and $y$ are said to be adjacent. We say that a graph $G^{\prime}=\left(X^{\prime}, E^{\prime}\right)$ is a subgraph of a graph $G=(X, E)$ if $X^{\prime} \subset X$ and $E^{\prime} \subset E$. A graph is said to be complete if $E=X^{(2)}$. A complete graph whose size is $n$ will be denoted by $K_{n}$. A complete maximal (in the sense of inclusion) subgraph of a graph $G$ is said to be a clique of $G$. A clique whose size is maximum is called a maximum clique [5]. To any vertex of a graph may be associated its neighborhood $\Gamma(x)=\{y \in X,\{x, y\} \in E\}$. The degree of a vertex $x$ is the cardinality of the set $\Gamma(x)$. If any vertex in the graph has the same degree, the graph is said to be regular.

A hypergraph $H$ [4] on a set $X$ is an ordered pair $(X, E)$ where $E$ is a set of nonempty subsets of $X$ such that $\bigcup_{e \in E} e=X$. Elements of $X$ are called vertices, those of $E$ hyperedges. The size of a hypergraph is the cardinality of its set of vertices. The line-graph of a hypergraph is defined as the graph whose set of vertices are the hyperedges of the hypergraph and where there is an edge between two vertices if the associated hyperedges have a nonempty intersection. To any graph may be associated its neighborhood hypergraph defined by: $\left(X,(\{x\} \cup \Gamma(x))_{x \in X}\right)$. Moreover, we will say that the hyperedge $\{x\} \cup \Gamma(x)$ is generated by the vertex $x$ and $x$ will be called source pixel of $\{x\} \cup \Gamma(x)$.

A digital image $\mathcal{I}\left[11\right.$ is a map from a subset $X$ (generally finite) from $\mathbb{Z}^{2}$ in a subset $\mathcal{C}$ of $\mathbb{Z}^{n}$. Elements of $X$ are called points, those of $\mathcal{C}$ colors. A couple $(x, I(x))$ where $x$ belongs to $X$ is called a pixel. However, the confusion between a point $x$ and $(x, I(x))$ is often made and we will not depart from it since it keeps its meaningfulness. A tiling (or a tessellation) of $\mathbb{R}^{2}$ [19] is a partition of $\mathbb{R}^{2}$. The tilings generally studied are constrained by a limited number of geometric configurations called tessels. Given a tiling, the choice of an arbitrary point in the tessels, and the fact to link two points if the tessels share a common side allows to build a mesh. For the case of regular tilings where the tessels are regular polygons, the center of gravity of the polygon is often chosen and therefore leads to a regular mesh. In image processing, three types of meshes are used: hexagonal, triangular and square ones. Because of the current technological devices and the natural data structuring, the last type is the most used and we will therefore restrict our study to this kind of mesh. If a distance on $\mathbb{Z}^{2}$ defines a undirected, simple, loopless and regular graph on a mesh, that distance will be called a grid distance. A grid is then a nonempty set of $Z^{2}$ with an associated grid distance. On square grids, two distance are mainly used: the city block (or 
square) distance for which a given pixel has four neighbors and the chessboard (or diamond) distance for which a given pixel has eight ones. This paper will only deal with the chessboard distance.

\section{Image Neighborhood Hypergraph Modeling}

\subsection{Spatiocolorimetric Neighborhood of a Pixel}

Let $d$ be a distance on the set of colors $\mathcal{C}$ and $d^{\prime}$ be a distance that defines a grid on $X \subset \mathbb{Z}^{2}$. Let $\alpha$ and $\beta$ be two strict positive reals. A unique neighborhood $\Gamma_{\alpha, \beta}(x)$ for the digital image $\mathcal{I}$ may be associated to any pixel $x$ of $X$ by:

$\Gamma_{\alpha, \beta}(x)=\left\{y \in X, y \neq x\right.$ such as $d(\mathcal{I}(x), \mathcal{I}(y))<\alpha$ and $\left.d^{\prime}(x, y)<\beta\right\}$

We will call $d^{\prime}$ grid distance and $d$ colorimetric distance. The definition of colorimetric distances highly correlated with the perception of the human brain is always nowadays an extremely delicate domain of study of the neuroscientific field. However we will assume that such functions exist even if the ones we will use may not appear appropriate with our sensations at the sight of an image. Looking at the previous definition, it appears that the first part of it defines a neighborhood in the space $\mathcal{C}$ whereas the second one only involves the spatial domain. We will therefore respectively call $\alpha$ and $\beta$, colorimetric threshold and spatial threshold. Moreover we will qualify $\Gamma_{\alpha, \beta}(x)$ of spatiocolorimetric neighborhood. That notion allows to describe some consistency or homogeneity of a pixel with its environment. It is also interesting to see that such a neighborhood has a useful property: it increases in the sense of inclusion both with $\alpha$ and $\beta$. For instance, if we chose two colorimetric thresholds $\alpha$ and $\alpha^{\prime}$ such as $\alpha<\alpha^{\prime}$, for a fixed $\beta, \Gamma_{\alpha, \beta}(x) \subseteq \Gamma_{\alpha^{\prime}, \beta}(x)$. That is due to the fact that if a pixel $y$ is, from the color point of view, close of $x$ at $\alpha$, it remains close to $x$ at $\alpha^{\prime}$. This argument is the same if we chose the spatial thresholds and it is possible to show it also for them. The main interest of that property is that it implies a certain regularity in the modeling that we will now present.

\subsection{Image Neighborhood Hypergraph}

It is now possible to define an image neighborhood hypergraph (INH) $\mathcal{H}_{\alpha, \beta}$ on $X$ by $\mathcal{H}_{\alpha, \beta}=\left(X,\left(\{x\} \cup \Gamma_{\alpha, \beta}(x)\right)_{x \in X}\right)$. It is useful to precise that such a definition is correct since any hyperedge $\Gamma_{\alpha, \beta}(x)$ is nonempty: it contains at least the pixel $x$. Moreover the union of all the hyperedges is $X$ itself. We will call $\Gamma_{\alpha, \beta}(x)$ hyperedge centered in (or generated by) the pixel $x$. As it directly inherits from the spatiocolorimetric neighborhood definition, an INH also increases with $\alpha$ and $\beta$ in the sense of the hyperedges inclusion. The figure 1 shows a part of an INH and its associated line-graph for a spatial threshold $\beta$ of 1 for the chessboard distance. For this figure we did not precise the colorimetric distance and its associated threshold as that is not relevant. However the first noticing is that displaying hypergraphs structures in this way leads to a muddled interlacing of patterns of various shapes and colors. 

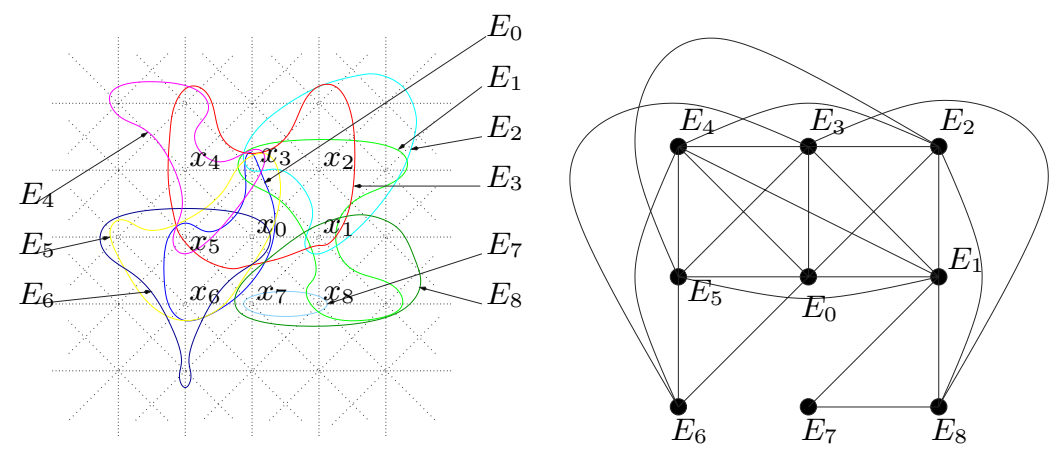

Fig. 1. A part of an image neighborhood hypergraph and its associated line-graph.

\section{First Attempts of Representation}

In this section we will describe the difficulties we encountered with our first attempts to represent the image neighborhood hypergraph structure in a simple bidimensional way as in the figure 1] That simple idea consisted in associating to represent hyperedges by the set of pixels that they contained.

\subsection{Coloring Hyperedges}

The first idea in representing an INH will probably be to associate a color to any hyperedge of it. Moreover it is then natural to color them in such a way that two intersecting hyperedges will have different colors. In order to make the representation as simple as possible it should be interesting to have the least number of colors. Generally speaking, this problem is known as the hyperedge coloring of a hypergraph $H$ and that minimum number of colors associated with it is called the chromatic index of $H$, denoted by $q(H)$. That problem relies on the chromatic number of a graph as lemma 1 precises it. The chromatic number of a graph is the minimum number of colors that are needed to color the vertices of that graph in such a way such that two adjacent vertices have distinct colors [1].

Lemma 1. The chromatic index $q(H)$ of a hypergraph $H$ is also the chromatic number $\chi(L(H))$ of its associated line-graph $L(H)$.

$$
q(H)=\chi(L(H))
$$

Proof. The proof directly comes from the definitions. Two intersecting hyperedges will have distinct colors and therefore their associated vertices in the linegraph of the hypergraph will also have different colors. That fact allows us to claim that necessarily $q(H) \leq \chi(L(H))$. Conversely two distinct vertices of the line-graph with different colors ensures that their associated hyperedges will also have different colors and therefore that $\chi(L(H)) \leq q(H)$. Hence we can conclude to the equality of both quantities $\chi(L(H))$ and $q(H)$. 
Unfortunately computing the chromatic number of a graph is generally not an easy thing... it is NP-hard [15]. Nevertheless we can particularize it to our line-graphs. The worst case that may appear is the one such that any hyperedge of the hypergraph has maximal cardinality, that is, when only the spatial threshold is discriminating. In this case, on the 8-connected grid, for a fixed threshold $\beta$, a pixel generates a square hyperedge whose cardinality is $(2 \beta+1)^{2}$ (we will assume that there are no border problems if we suppose that the image loops itself). It is easy to see that the resulting line-graph is regular: as the same hyperedges configuration appears on the whole image, the number of intersections is the same for every vertex of the graph. Remark that, in the case of a regular graph, computing the chromatic number is as hard as in the general case [17. However it is possible to represent the vertices of the line-graph by the pixel that generated the corresponding hyperedge and it is clear that any vertex-pixel of a fixed hyperedge $e$ (that we will call as the "initial" hyperedge) is the center of a hyperedge $e^{\prime}$ that intersects with $e$. This is the case of the pixel we called "member" in the figure 2. But these hyperedges are not the only ones that intersects $e$. This is the case for instance for the pixel that we called "external" on the figure 2 It is however easy to see that no pixel further than $2 \beta$ from the "initial" pixel will generate a hyperedge that intersects with the initial hyperedge. Hence we can precisely give the number of intersections that a hyperedge have in the INH: $(4 \beta+1)^{2}-1$ (if we assume that a hyperedge does not intersect with itself). The degree of a vertex in the line-graph is $(4 \beta+1)^{2}-1$ (see Fig. 3).

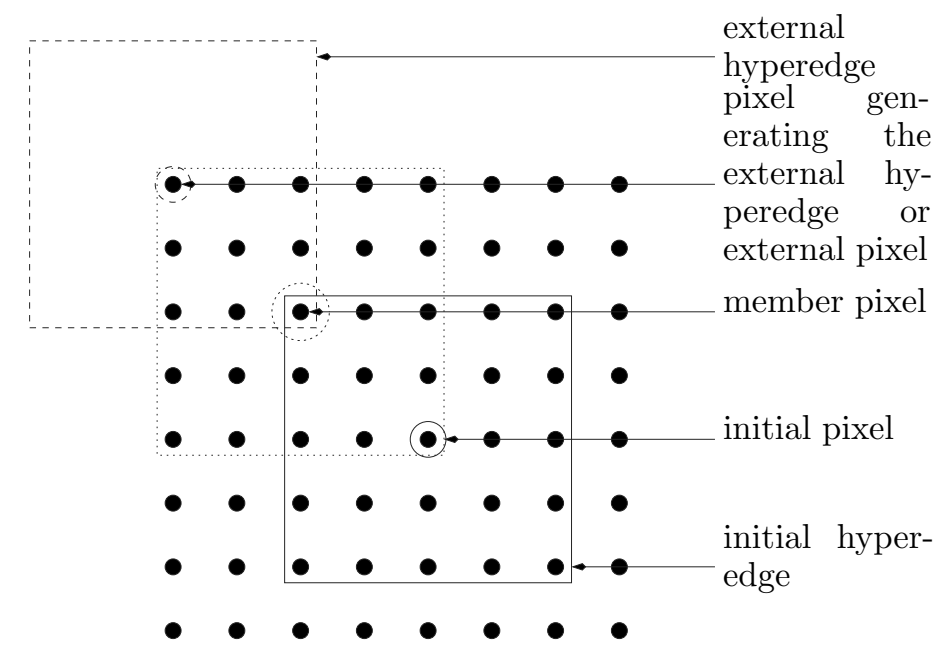

Fig. 2. Determination of the worst case for the chromatic index in a image neighborhood hypergraph. We display in this the case for $\beta=2$

Lemma 2. In the line-graph $L\left(\mathcal{H}_{\star, \beta}\right)$ of an image neighborhood hypergraph $\mathcal{H}_{\star, \beta}$, the degree of any vertex is $(4 \beta+1)^{2}-1$. 
Computing the chromatic number of that graph is similar to determining the size of the largest clique in it [1]. As we represent the vertices of the line-graph by pixels on a grid, we can make some claims from the geometrical point of view to determinate the size of this clique. Let us take a vertex $o$ (associated to a pixel $O)$ in the line-graph. There is at least one clique $C$ that lies in the neighborhood of $o$ in the graph. Using the representation of the line-graph that we previously defined, we may see the pixel $O$ as a "central" one (in the sense of geometrical central symmetric) for the clique and we will say that the clique $C$ is centered in $o$. Therefore if we take another vertex $p$ in the line-graph that belongs to the neighborhood of $o$ and if we consider the pixel $P$ associated with it, we can conclude that the symmetric $Q$ of $P$ is associated to a vertex $q$ that also lies in the neighborhood of $o$ and therefore belongs to the same clique. Moreover we may apply the same arguments to any vertex associated with a pixel which is at the same distance of $P$ from $O$ thanks to isometric transformations of the discrete plane $[25,20]$. Then we can assert the following result:

Lemma 3. If a pixel $P$ such that $d^{\prime}(O, P)=\beta_{0}$ is associated to a vertex $p$ that belongs to a clique $C$ centered in o, then the set of pixels

$$
\left\{P^{\prime} \in X \text { such that } d^{\prime}\left(O, P^{\prime}\right)=\beta_{0}\right\}
$$

is associated with vertices of the graph that also belong to $C$.

Under the previous assumptions and notations, we may also say that any pixel located in the disk whose center is $O$ and whose radius is $\beta_{0}$ is also associated with vertices that also belong to the clique. That fact is simply due to the definition of our worst case as any hyperedges are of size $\beta$ and a triangular inequality argument. The idea is now to determinate precisely the maximal size of that disk.

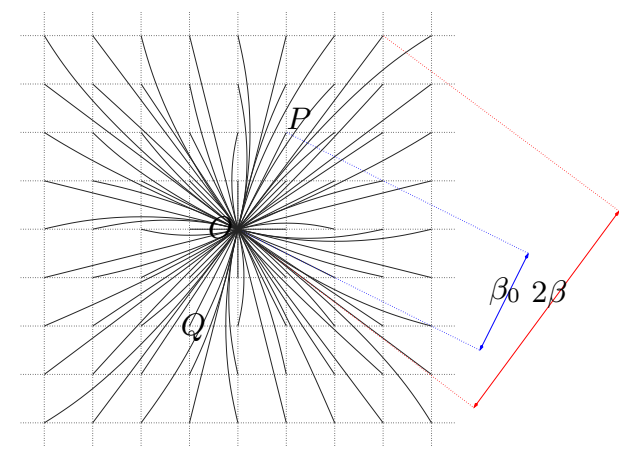

Fig. 3. Illustration of some geometric remarks concerning hyperedges coloration. Example of a partial line-graph built with $\beta=2$

Assume that such a disk $D$ exists. Let us call its center $O$ and its radius $\beta_{0}$. Let us take a pixel $P$ at $\beta_{0}$ from its center. It is clear that $P$ belongs to 
$D$. Its symmetrical $Q$ around $O$ also belong to $D$. If we want $P$ and $Q$ to be respectively associated with vertices $p$ and $q$ that belong to the clique, necessarily $P$ and $Q$ are linked by an edge in the line-graph and therefore each lies in the neighborhood of the other one if the line-graph. Moreover the distance between $P$ and $Q$ is simply $2 \beta_{0}$ and cannot exceed $2 \beta$ (as we already prove it). Therefore we have to solve the equation:

... whose solution is simply:

$$
\arg \max _{\beta_{0} \in \mathbb{N}} \quad 2 \beta_{0} \leq 2 \beta
$$

$$
\beta_{0}=\beta
$$

The maximal radius of the disk we sought is then $\beta$ and we can give the following lemma:

Lemma 4. The upper bound for the size of the maximum clique of a line-graph associated with an image neighborhood hypergraph $\mathcal{H}_{\star, \beta}$ is $(2 \beta+1)^{2}$.

In the worst case i.e. when the colorimetric threshold is not discriminating, such a size is reached.

So, for instance, for an INH built for $\beta=2$, the representation of its hyperedges already leads to a 25-colored muddle. That quadratic increase of the number of colors is unfortunately not the only problems we were confronted to represent the hyperedges of an INH.

\subsection{Other Representation Problems}

Hyperedges Connectivity. On the 8-connected grid, let us consider two hypergraphs representations built for $\alpha=5$ on the subimage of the figure 4 and the colorimetric distance simply compares the absolute value of the difference between the colorimetric value of the pixels with $\alpha$. For $\beta=1$, the hyperedge generated by the "central" pixel is represented in blue, for $\beta=2$, in red. In order not to overload that figure, the superimposition of the two hyperedges has not been explicitly shown but it must be clear that due to the increasing property of the INH, the blue hyperedge is contained in the red one. What is remarkable in that figure is that such a representation of hyperedges may lead to the appearance of disconnected areas in it and holes in hyperedges increasing the difficulty of the reading for a potential user.

Which Curves to Chose? As there are no convexity properties for the hyperedges (see figure 4), our initial choice of curves of the splines family did not appeared as very relevant. Moreover, after coloration and connectivity difficulties, the problem of the choice of the control points of these curves was added. The representation of the hypergraph structure itself appeared us then so tricky that we chose to go towards another representation of the structure: the one of its line-graph. 


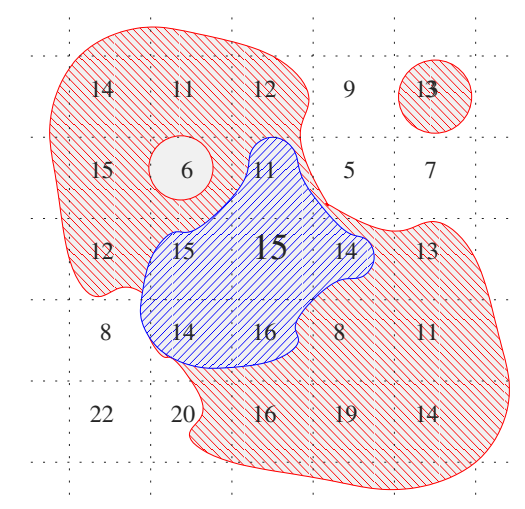

Fig. 4. In an INH, hyperedges are not necessarily connected in the sense of the underlying grid.

\section{Line-Graph Representation of an INH}

Our main motivation with that representation was not to display a particular graph structure: we had the intention to make it interactively investigational by a potential user. It is certainly of great interest for the one interested in discovering our modeling to chose himself his own point of view on the structure and, better, in allowing him to navigate through the structure itself. We develop such a tool and in investigating the structure we were able to characterize image phenomena such as noise or object edges lying inside an image [10,22.

\subsection{Image Correlation}

Our first concern was to strongly correlate our representation with the data it came from. That is why we chose to display the original image on the representation. In order not to disturb the visualization of the graph structure, we chose to draw it with a parametric opacity. As any hyperedge is generated by a pixel of the digital image, it is natural to represent it by a point or better by a polyhedron or a sphere located at the vertical of the pixel. The elevation of the center of gravity of that polyhedron from the source pixel is controllable and may moreover be proportional to the intensity of the source pixel giving the representation a tridimensional aspect that makes easier its reading. To show the size of the hyperedge we make its radius proportional to the cardinality of the hyperedge (that proportional factor is also controllable).

\subsection{Line-Graph Structure Representation}

The line-graph is not a planar graph. For $\beta=1$ for instance, we showed that the degree of its vertices may reach 24 and therefore it should not be rare to 

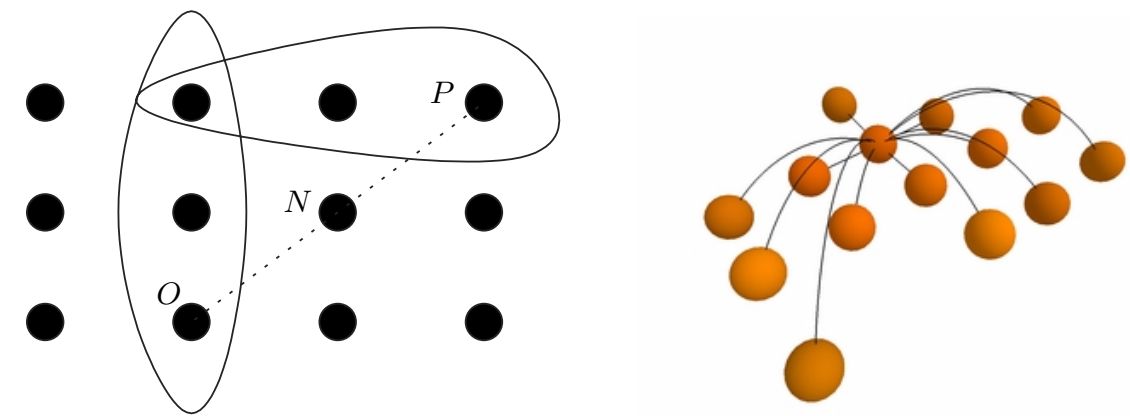

Fig. 5. The representation of the line-graph structure may gives bad impression. Some vertices may appear linked in the structure whereas they are not. The local display of a hyperedge of an image neighborhood hypergraph.
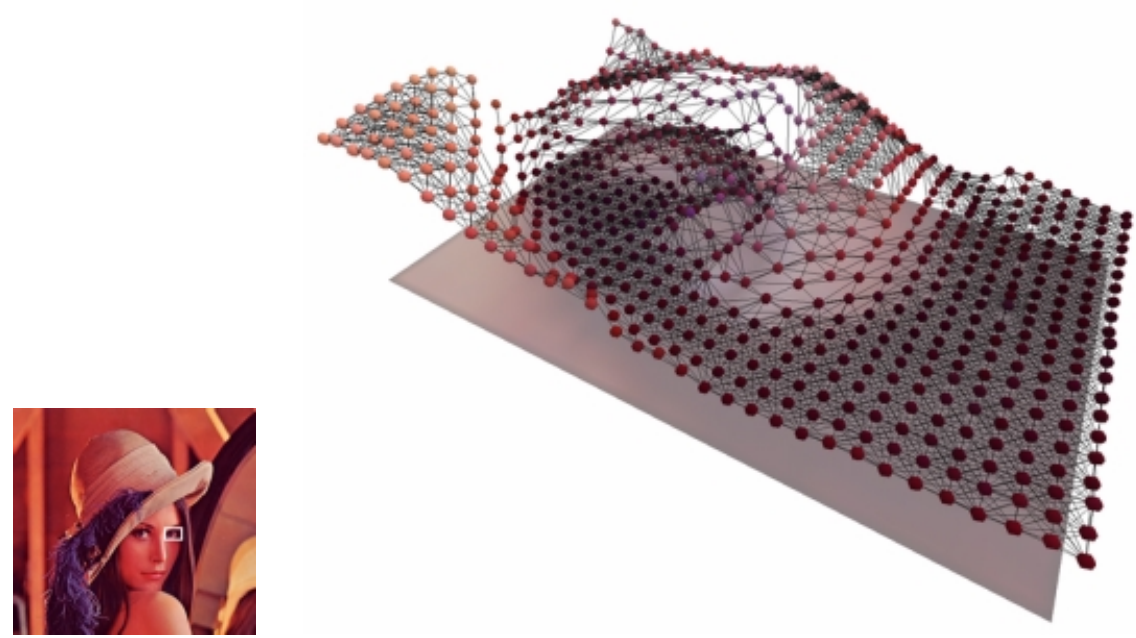

Fig. 6. Sample representations of the line-graph of an INH. The image is a RGB-color picture. For a pixel $x$, color is coded by $\left(x_{r}, x_{g}, x_{b}\right)$. The colorimetric distance used was $d(x, y)=\sqrt{\left(x_{r}-y_{r}\right)^{2}} \cdot \alpha$ was set to 25 and $\beta$ to 1 . Note the weak degree of some vertices in that graph: that allowed us to partially characterize edges of objects in an image [10].

see $K_{5}$ or $K_{3,3}$ in that graph 11. However we chose to link the vertices of the graph by simple segment lines even if it could give bad impression on the graph. From the figure 5 for instance, two hyperedges are explicitly drawn. They are generated by the pixels $O$ and $P$. They intersect and therefore a link between $O$ and $P$ must be shown. Let us assume that the hyperedge generated by $N$ is reduced to $N$ itself. The user may therefore have the (bad) impression that $N$ is linked with both $P$ and $O$ as $N$ lies between these two points. Firstly it must be clear that such cases may be corrected by the user himself. As the elevation 

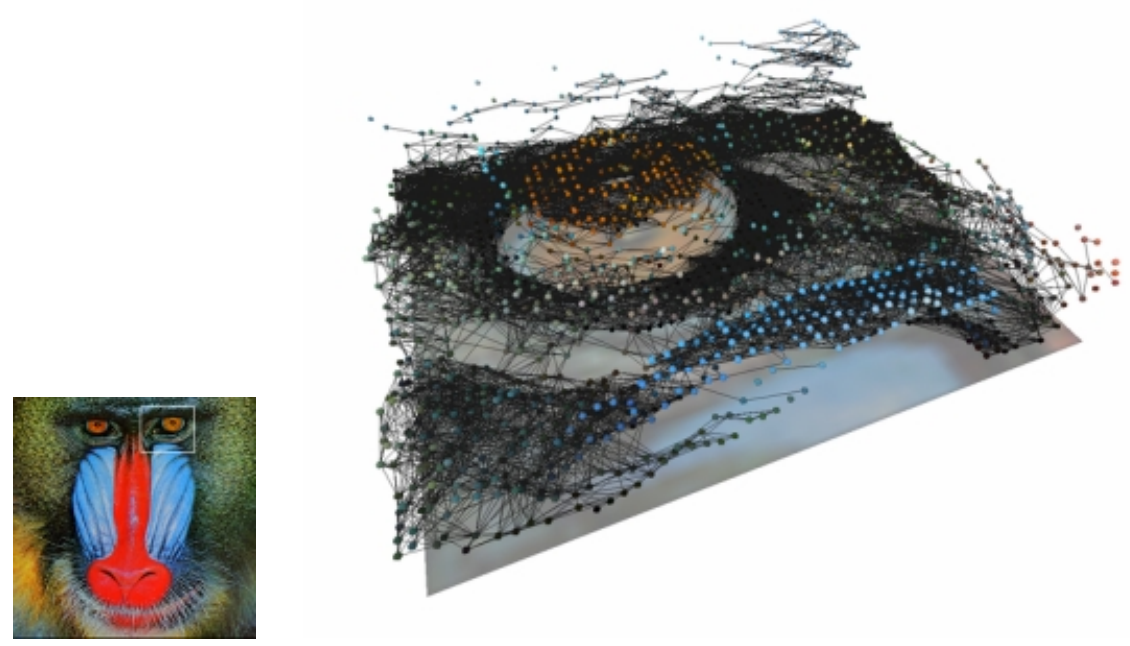

Fig. 7. Sample representations of the line-graph of an INH. The image is a RGB-color picture. For a pixel $x$, color is coded by $\left(x_{r}, x_{g}, x_{b}\right)$. The colorimetric distance used was $d(x, y)=\max \left\{\left|x_{r}-y_{r}\right|,\left|x_{g}-y_{g}\right|,\left|x_{b}-y_{b}\right|\right\} . \alpha$ was set to 20 and $\beta$ to 2 . Note that some areas are disconnected from the rest of the graph, some vertices are also completely isolated from it. That allowed us to characterize noise in an image [7 8|9|22].

of the polyhedron representing the hyperedge generated by $N$ is controllable by the user. As its cardinality is 1, its intensity will probably different enough from its neighbors and therefore its elevation may also be different enough in order not the segment line not to cross the polyhedron. Moreover, as the previous argument may not appear as sufficient, we give the possibility to the user to individually select a polyhedron and to display the corresponding hyperedge. Only the pixels belonging to the hyperedge are drawn and moreover in order not to give also bad impression on that representation, parabola arcs are drawn if the pixels are located are located at more than 2 units (in the sense of the grid distance) from the source pixel (see Fig. 51).

\subsection{Current Restrictions}

The main problem with this kind of representation is that it is not possible, at the moment, to display too large images. For instance the figures 6 and 7 only involves $33 \times 21$ and $55 \times 46$ color images. The main drawback is the memory cost of the structure and the slowness of the current video cards 1 . However, at the sight of the progress recently made in that technical field, we do not despair to display larger structures in a near future. Moreover as it is possible to deal

\footnotetext{
${ }^{1}$ For the figures 6 and 7 the construction and display have been made on a $1.2 \mathrm{MHz}$ Athlon GeForce2 MX video card. Navigation through the structure is very flowing but begins to be difficult when the size of the image reaches more than 50000 pixels.
} 
with sub-images and parallelize processes, it should be possible to build subrepresentations of the image and to deal only with the nearest of them when the display is made.

\subsection{Characterizing Regions of Interest in an Image}

However, at the sight of pictures resulting of the display, it is interesting to note that regions of interest appear from the graph representation. For instance, weakly connected vertices appear in the left upper part of figure 6 characterizing edges of objects or of homogeneous areas in the original image [10]. Totally disconnected vertices, small sets of them or very weak connections between a set of vertices and larger size areas of the graph as shown in figure 7 allowed us to give non-statistically based definitions of noise in an image $7 \mathbf{7 8 9 2 2}$.

\section{References}

1. B. Bollobás Modern Graph Theory, Springer-Verlag, (1998).

2. C. Berge, "Introduction à la théorie des hypergraphes", Les Presses de l'Université de Montréal, (1973)

3. C. Berge, "Graphs". North Holland, (1987).

4. C. Berge, "Hypergraphs". North Holland, (1989).

5. I. Bomze, M. Budinich, P. Pardalos, and M. Pelillo, The maximum clique problem, http://www.dsi.unive.it/ pelillo/papers/tr99-1.ps.gz, (1999).

6. A. Bretto, J. Azema, H. Cherifi, and B. Laget, Combinatorics and image processing in "Computer Vision Graphic in Image Processing", 59, (5), September, (1997) $265-277$.

7. A. Bretto, and H. Cherifi, A noise cancellation algorithm based on hypergraph modeling in "IEEE Workshop on Digital Signal Processing" (1996) 1-4.

8. A. Bretto, and H. Cherifi, Noise detection and cleaning by hypergraph model in "IEEE Computer Sciences" International Symposium on Information Technology: Coding and computing, IEEE Computer Sciences, (2000) 416-419.

9. A. Bretto and S. Chastel, A note on valued polyominoes hypergraph image modeling, International Journal of Computational and Numerical Analysis and Applications, to appear.

10. S. Chastel, A. Bretto, and H. Cherifi, Image neighborhood hypergraph model and edge detection in "JCIS Proceedings" 3, Association for Intelligence Machinery, (1998), 272-275.

11. J. P. Cocquerez and S. Philipp, Analyse d'images : filtrage et segmentation, Enseignement de la physique, Masson, (1995).

12. T.K. Dey, H. Edelsbrunner, S. Guha "Computational topology", Technical report, (1998).

13. R. Diestel, "Graph Theory", Graduate Text in Mathematics, Springer-Verlag, (1997).

14. M. Gondran, and M. Minoux, "Graphs and Algorithms" Wiley, Chichester, (1984).

15. I. Hoyler, The NP-completeness of edge coloring, SIAM J. Comput., 10: 718-720, (1981)

16. J. Jing-Ying, Multilevel Median Filter Based on Fuzzy Decision in "IEEE Trans. Image Processing", 4: 680-682, (1995) 
17. D. Leven and Z. Galil, NP-completeness of finding the chromatic index of regular graphs, J. Algorithms, 4: 35-44 (1983)

18. V.A. Kovalevsky, Finite topology as applied to image processing in "Computer Vision, Graphics, and Image Processing" 46 (1989) 141-161.

19. A. Montanvert, and J.M. Chassery, "Géometrie discrète en analyse d'images" Traité des Nouvelles Technologies. Série Images. Hermès, Paris (1991).

20. J.-P. Réveillès, Géométrie discrète, calculs en nombre entiers et algorithmique, Thèse de doctorat, Université L. Pasteur, Strasbourg, (1991).

21. F.P. Preparata, and M.I. Shamos, "Computational Geometry : An Introduction", Springer, New York, (1985).

22. S. Rital, A. Bretto, H. Cherifi, and D. Aboutajdine, Modélisation d'images par hypergraphe. Application au débruitage, ISIVC Proceedings (ed. IAPR), Rabat (2000), 25-34.

23. A. Rosenfeld, "Digital Image Processing", Academic Press, San Diego, (1982)

24. J.C. Russ, "The image processing handbook" CRC press, IEEE press, Springer, Berlin (1999)

25. K. Voss, Discrete Images, Object and Functions in $\mathbb{Z}^{n}$, Springer-Verlag (1993) 